\title{
THE OWENS VALLEY MILLIMETER ARRAY
}

\author{
N. SCOVILLE, J. CARLSTROM, S. PADIN, A. SARGENT, S. SCOTT, \\ \& D. WOODY \\ Owens Valley Radio Observatory, California Institute of Technology, Pasadena \\ CA, USA 91125
}

\begin{abstract}
The present status and projected two year developments for the Owens Valley Millimeter Array are described and a brief summary of the astronomical research is presented.
\end{abstract}

\section{INTRODUCTION}

The Owens Valley Millimeter Array is an aperture synthesis array consisting of $10.4 \mathrm{~m}$ diameter telescopes (cf. Padin et al. 1991). Originally built as a three element array, it is currently undergoing a major expansion to six telescopes with funding provided jointly by Caltech, the National Science Foundation, and the K.T. and E.L. Norris Foundation. The array is located in a semi-desert region 250 miles north of Los Angeles on the east side of the Sierra Nevada range, near Bishop, California. The elevation $(1200 \mathrm{~m})$ and dry climate permit observations at wavelengths as short as $1 \mathrm{~mm}$ (Scott \& Woody 1991). At present the array is operated with dual frequency SIS receivers for the $\lambda=2.6$ and $1.3 \mathrm{~mm}$ bands.

Major upgrades now in progress include the addition of the three new telescopes, development of sideband separation receivers to improve overall sensitivity, a broadband continuum correlator, and a real-time relational database. The schedule for integrating the new telescopes into the array is: \#4 (December 1992), \#5 (February 1993), and \#6 (December 1993). Funding is currently being sought by a consortium of Canadian universities to add a seventh telescope and to double the array baseline.

\section{TELESCOPES AND BASELINE}

The $10.4 \mathrm{~m}$ diameter telescopes (Leighton 1978) utilize steel Alt-AZ mounts, steel space-frame backstructures and aluminum honey-comb panels. The telescopes have surface errors of about $35 \mu \mathrm{m} \mathrm{rms}$ at night and $50 \mu \mathrm{m}$ rms under solar illumination. The measured aperture efficiencies at $\lambda=2.6$ and $1.3 \mathrm{~mm}$ are 0.65 and 0.45 respectively. At $\lambda=2.6 \mathrm{~mm}$, the gain-elevation effect over the full elevation range is less than $6 \%$ with a fixed focus position; there is less than $20 \mu \mathrm{m}$ rms deviation from homology due to gravitational deflections over the elevation range from 30 to $75^{\circ}$ (Woody 1990). The absolute pointing accuracy of the telescopes is $\sim 6^{\prime \prime} \mathrm{rms}$ with some larger deviations at sunrise and sunset. The nighttime tracking accuracy is better than $3^{\prime \prime}$ rms. 
The telescopes can be positioned at any of 25 stations laid out on a track with an inverted $\mathrm{T}$ geometry ( $200 \mathrm{~m}$ east-west and $220 \mathrm{~m}$ north-south). Telescopes are moved between stations by a transporter which sits on the track. These moves typically require one hour per telescope. The basic station spacing increment near the $T$ intersection is $10 \mathrm{~m}$, but a station is provided at $65 \mathrm{~m}$ west to allow for 5-m increments. The array has a maximum resolving power of $\sim 2^{\prime \prime}$ at $\lambda=2.6 \mathrm{~mm}$ and $\sim 1^{\prime \prime}$ at $\lambda=1.3 \mathrm{~mm}$. To obtain images with this resolution requires 12-20 baselines depending on the source declination.

\section{RECEIVERS}

Each telescope is equipped with a $4.5 \mathrm{~K}$ cryostat at the Naysmith focus. The cryostats can accommodate four SIS mixers and are presently configured with one 80 - $115 \mathrm{GHz}$ mixer and one 215 - $270 \mathrm{GHz}$ mixer (Woody, Giovanine \& Miller 1989). New optics have been installed to permit rapid switching between bands in order to exploit the best weather conditions and enable phase calibration at $\lambda \cong 3 \mathrm{~mm}$ (where the system sensitivity is optimal) during $\lambda \sim 1$ $\mathrm{mm}$ observations. The optics allow simultaneous observations in both bands by replacing a mirror with a polarying grid.

The cryostats contain closed-cycle refrigeration systems that use a commercially available $15 \mathrm{~K}$ refrigerator and a Joule-Thompson expansion loop. The $\mathrm{J}-\mathrm{T}$ throttle is a needle valve which can be adjusted from outside the cryostat; the flow rate set is a compromise between cooling capacity and temperature. The cooling capacity of the J-T system is $\sim 1 \mathrm{~W}$ with a J-T return pressure of 16 psi absolute.

The mixers use a quarter-height rectangular waveguide structure with a corrugated feedhorn and a single, adjustable backshort. The feedhorn provides a Gaussian beam pattern with a full width of $30^{\circ}$. A $\lambda / 4$ transition is used to match the circular-waveguide output from the feedhorn to the reduced-height rectangular guide in the mixer block. An SIS junction $\left(\mathrm{Nb}-\mathrm{AlO}_{x}-\mathrm{Nb}\right)$, fabricated on a quartz substrate, is mounted across the center of the guide parallel to the E-field for the $\mathrm{TE}_{10}$ mode. The backshort is a choked, noncontacting structure, with a stepper motor for remote computer-controlled tuning. The single backshort tuner allows the mixers to be tuned single-sideband for the lower sideband, but it is not usually possible to significantly favor the upper sideband. The mixers require less than $1 \mu \mathrm{W}$ of $\mathrm{LO}$ power; this is coupled via $\mathrm{a} \sim 1 \%$ dielectric beamsplitter outside the cryostat.

The $\lambda \sim 2.6 \mathrm{~mm}$ receivers typically provide single sideband system temperatures, including the effects of the atmosphere, of $\sim 150 \mathrm{~K}$ at $100 \mathrm{GHz}$ and $\sim 400 \mathrm{~K}$ at $115 \mathrm{GHz}$. The latter frequency is dominated by the atmospheric opacity in the wings of the $118 \mathrm{GHz} \mathrm{O}_{2}$ line. At $\lambda \sim 1.3 \mathrm{~mm}$ the system temperatures are presently $\sim 600 \mathrm{~K}$; but should improve with the installation of new mixers this winter.

The local oscillator for each receiver is provided by a mechanically tuned InP Gunn oscillator of the type described in Carlstrom, Plambeck \& Thornton (1985). For the $3 \mathrm{~mm}$ band an oscillator with a single mechanical tuner covers 82 - $115 \mathrm{GHz}$. For the $\lambda \sim 1.3 \mathrm{~mm}$ band, a high power, two tuner oscillator feeds a tripler to provide LO power over the range $208-270 \mathrm{GHz}$. The oscillators are 
phase locked using the arrangement described by Padin, Woody \& Scott (1988). This LO system results in an inter-receiver phase jitter of $7^{\circ}$ rms at $115 \mathrm{GHz}$, corresponding to a sensitivity degradation of $0.8 \%$. The long-term stability of the telescope-based components is $60^{\circ}$ peak-to-peak in 24 hours at at $115 \mathrm{GHz}$. These phase drifts are caused primarily by temperature variations in the coaxial line which carries the reference signal to each telescope.

\section{IF SYSTEM AND DELAY LINES}

The first IF stage is a cooled HEMT amplifier with a 1-2 GHz bandwidth, (Padin 1991). The amplifier is a balanced circuit in which identical HEMT devices and matching circuits are fed by a quadrature hybrid. Each matching circuit tranforms the $50 \Omega$ input impedance to the optimum noise match for the HEMT. This yields a minimum noise temperature, but the power match at the input of the matching circuit is poor. The beauty of balanced configuration, however, is that the power match at the hybrid input is determined by the symmetry of the two amplifier circuits and not by their input match. Consequently, the balanced circuit can yield excellent noise performance with a well-matched input using amplifiers with no feedback. The HEMT amplifier has a noise temperature of $\sim 6 \mathrm{~K}$ from $1-2 \mathrm{GHz}$ and contributes $\sim 24 \mathrm{~K}$ when referred to the mixer input.

For the tracking delay, a wideband $(0.5-3.5 \mathrm{GHz})$ system has recently been developed. For the three longest segments $(512,256$ and $128 \mathrm{~ns})$ the delays are realized in optical fiber; the remaining shorter delay segments are coaxial and microstrip transmission lines (cf. Padin \& Soares 1992).

\section{CORRELATORS}

The array has two correlator systems: a wideband analog cross correlator for continuum observations and a digital cross correlator for spectal-line observations (Padin et al. 1992). The continuum correlator operates at $1.5 \mathrm{GHz}$ and has a bandwidth of $1 \mathrm{GHz}$. It uses quadrature hybrids and double-balanced mixers to provide a complex multiplier for each baseline.

The digital cross correlator computes the real cross-correlation function for signals in four frequency bands, each up to $128 \mathrm{MHz}$ wide. These bands can be independently positioned anywhere in the 1 to $2 \mathrm{GHz}$ IF band. The IF is sampled at the Nyquist rate ( $u p$ to $256 \mathrm{MHz}$ ) and digitized into two-bits. The correlator has a serial architecture with the digitizers and multipliers always operating at the Nyquist rate for the selected bandwidths. Although this scheme underutilizes the processing power of the correlator when the digitizer input bandwidth is less than the maximum, it has the advantage of simplicity.

The correlator is divided into modules containing 128 lags (64 positive and 64 negative). For each baseline, four correlator modules can be associated with independent bands or cascaded to give different resolution options. Four bandwidths $(128,32,8$, and $2 \mathrm{MHz})$ and seven shift clock rates $(256,128,64,32,16$, 8 , and $4 \mathrm{MHz}$ ) are available.

The high-speed sections of the correlator are realized on a custom ECL gate array. Each gate array (2500 equivalent gates) contains the circuits for 8 lags along with switches to allow selection of different clock and signal sources. 
The high-speed clocks are transmitted differentially from one integrated circuit to the next while the slower data are single-ended. Integration is performed in CMOS gate arrays, each containing 3218 -bit synchronous counters.

The correlator uses 3-threshold digitization with 2-bit encoding appropriate for the multiplication schemes described by Cooper (1970); for the OVRO system the multiplication scheme with deleted inner products has been adopted. This results in a sensitivity of 0.88 relative to a perfect analog correlator. Digitization is performed by three high-speed ECL sampling comparators which detect transitions of the thresholds (Padin \& Ewing 1989). The transition rate for each threshold is monitored by a counter; actual digitizer thresholds are then computed so that digitizer-induced offsets and scale-factor errors can be corrected at the correlator output.

\section{ARRAY CONTROL SYSTEM}

The computer system that controls the array consists of a central VAX station 3100 connected to embedded processors in the telescopes and correlators. The VAXstation handles user interaction, general computing, low duty cycle array control, and disk storage. The embedded processors handle high duty cycle control such as telescope pointing, receiver control, and data integration. The microcomputers are microVAX II processors running $\mathrm{C}$ programs under the VAXELN operating system and connected to the VAXstation with Ethernet.

The key component in the array software is the Central Control Program which runs on the VAXstation (Scott \& Finch 1992). This program interprets and executes commands from interactive sessions and from submitted text files. Command interpretation and execution involves interaction with the embedded processors to coordinate tracking and integration. Data are assembled and written to the database disk on the SPARC II system (see below) by the Central Control Program. The interactive sessions may be from terminals located at the observatory, from remote sessions connected through the network, or from dial-in terminals and personal computers. Remote observing is fully supported and the array can be monitored from several locations simultaneously.

\section{DATA REDUCTION}

All engineering and astronomical data from the array are stored in a database where they are permanently on-line and immediately accessible to all users (Scoville et al. 1993). We have adopted a commercial relational database (Sybase) and the database server is run on a SPARC II. Information on different aspects of the system (eg. source positions, integrations, spectral lines, and spectrometer channels) are stored in tables which are then linked by common "key" columns. The relational database structure provides maximum flexibility for inquiries, since the "relationships" between data in the separate tables are postulated during data retrieval rather than during data storage. It also provides for minimum redundancy in data storage, maximizing the capability to keep a long span of data permanently on-line. Approximately 1 Gbyte of storage is required for each year of interferometer data and parallel copies of the full database are maintained at the Observatory and on campus. 


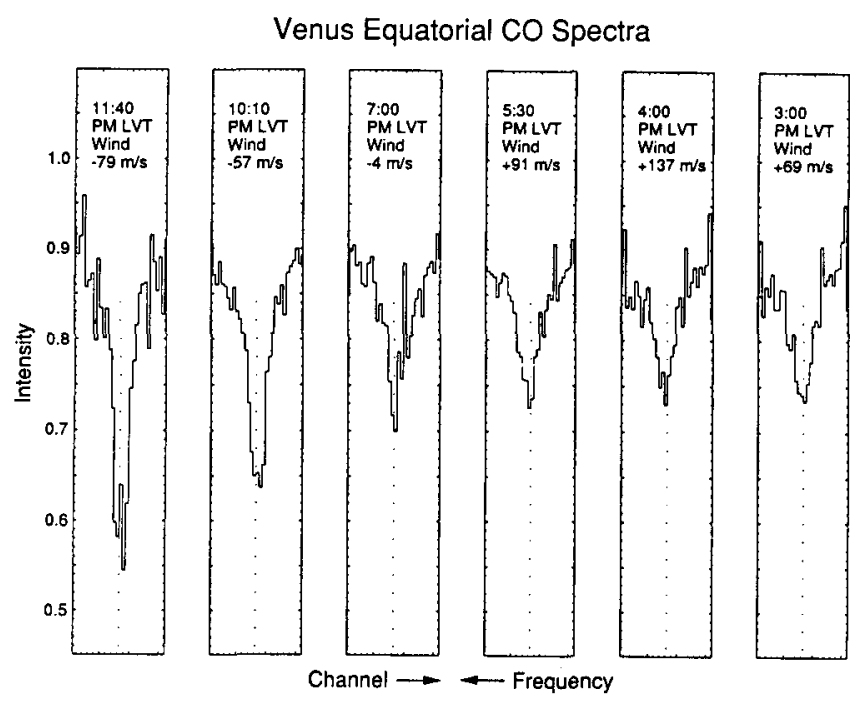

FIGURE I Spectra of CO absorption is Venus at $3^{\prime \prime}$ resolution show strong variations in the $\mathrm{CO}$ mixing ratio and Doppler shifts (each channel is $50 \mathrm{kHz}$ or $130 \mathrm{~m} \mathrm{~s}^{-1}$ ) - nightside red shifts and dayside blue shifts (Shah et al. 1991).

The ease of access to all interferometer data has enabled much better calibrations since observers can easily make use of each other's data for calibration purposes. The derived calibration information (eg. flux scaling constants and measured source fluxes) are also saved in the database.

The program used by astronomers to recall, analyze, calibrate, and display their data is a forms fill-in/mouse-driven program which contains a mixture of both fourth generation and standard $\mathrm{C}$ and Fortran routines. This program provides complete search and calibration capability and although the program is primarily designed to run interactively, it also permits automated, batch processing. The output of the program is calibrated FITS uv files which can be read directly by the NRAO AIPS package. Typically, the calibration of data for a single project can be accomplished in about half a day.

\section{ASTRONOMICAL HIGHLIGHTS}

The diversity of astronomical research possible with millimeter arrays is amply demonstrated by some of the projects carried out over the last five years with the Owens Valley array. This research spans the range from studies of planetary atmospheres, the mass-loss en velopes of evolved stars, protostars and protoplanetary nebula to galactic structure, nuclear starbursts, and ultraluminous high z galaxies. Highlight projects include:

- direct imaging of the $\mathrm{CO}$ absorption in Venus at $3^{\prime \prime}$ resolution which revealed 


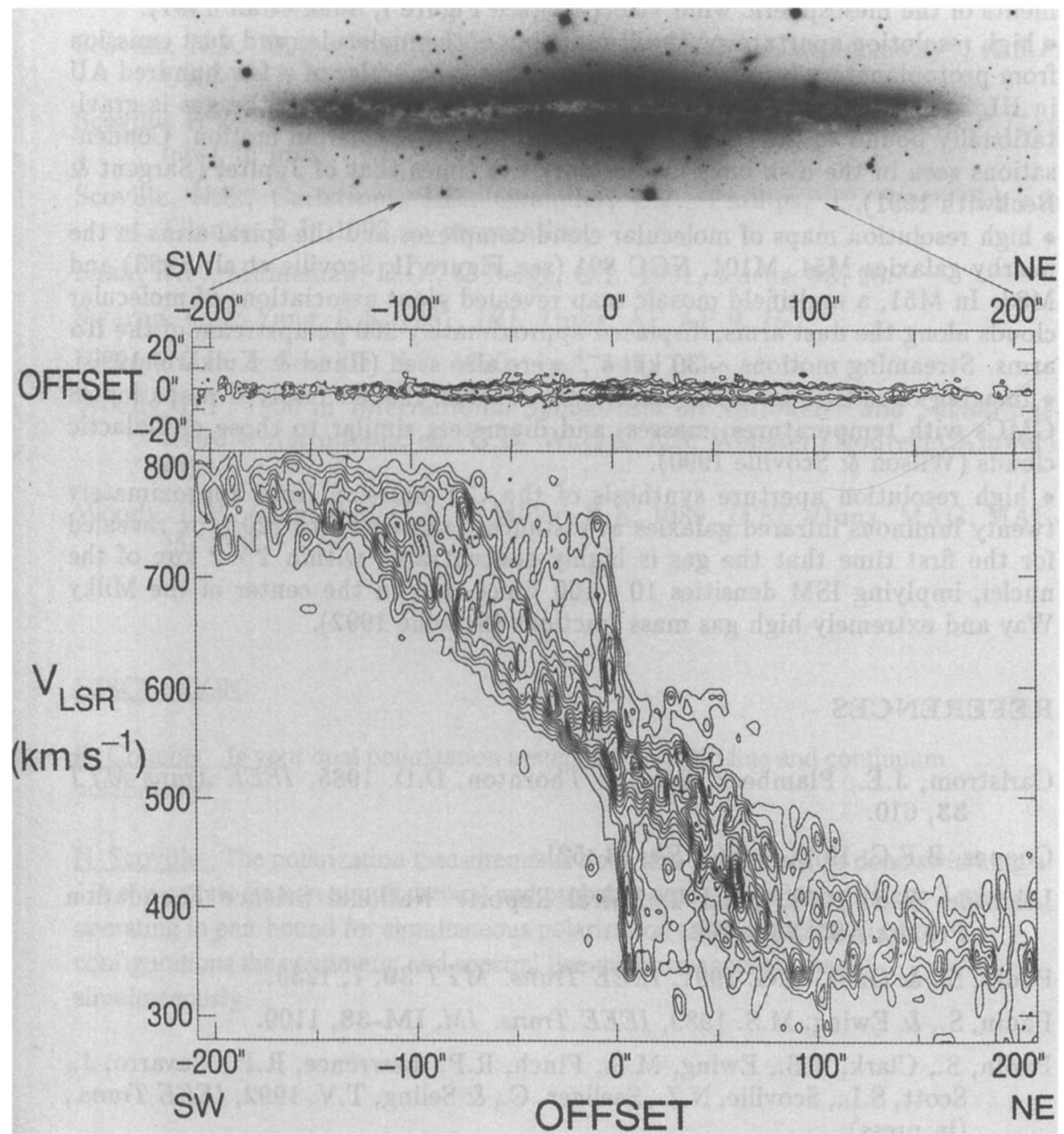

FIGURE II The CO (1-0) emission from NGC 891 at $2.3^{\prime \prime}(104 \mathrm{pc})$ resolution is shown together with an optical image (Scoville et al. 1993). In the middle figure the $\mathrm{CO}$ emission is shown as a function of longitude and latitude (integrated over the full range of galactic velocities); in the bottom panel the $\mathrm{CO}$ emission is shown as a function of longitude and velocity, integrated perpendicular to the galactic disk. Both representations of the $\mathrm{CO}$ data illustrate the major features of the gas distribution - central nuclear disk at less than $5^{\prime \prime}$ radius and a ring feature with maxima between 50 and $80^{\prime \prime}$ radius - extraordinarily similar to the $\mathrm{CO}$ distribution in the Galaxy. 
strong variations in the $\mathrm{CO}$ mixing ratio and provided the first global measurements of the mesospheric wind velocities (see Figure I, Shah et al. 1991).

- high resolution aperture synthesis mapping of the molecular and dust emission from protoplanetary/protostellar disk structures on scales of a few hundred AU in HL Tau, DG Tau, L1551, and IRAS 16293-24. In HL Tau the gas is gravitationally bound to the central star and orbiting in Keplerian motion. Condensations seen in the disk have masses only five times that of Jupiter (Sargent \& Beckwith 1991).

- high resolution maps of molecular cloud complexes and the spiral arms in the nearby galaxies M51, M101, NGC 891 (see Figure II, Scoville et al. 1993) and M83. In M51, a multifield mosaic map revealed giant associations of molecular clouds along the dust arms, displaced approximately $300 \mathrm{pc}$ upstream of the $\mathrm{H} \alpha$ arms. Streaming motions $\sim 30 \mathrm{~km} \mathrm{~s}^{-1}$ were also seen (Rand \& Kulkarni 1990). - imaging of individual molecular clouds in M33 yielded resolved maps for 38 GMCs with temperatures, masses, and diameters similar to those of Galactic clouds (Wilson \& Scoville 1990).

- high resolution aperture synthesis of the CO emission from approximately twenty luminous infrared galaxies at distances ranging up to $220 \mathrm{Mpc}$ revealed for the first time that the gas is highly concentrated within $1-2 \mathrm{kpc}$ of the nuclei, implying ISM densities 10 - 100 times that in the center of the Milky Way and extremely high gas mass fractions (Scoville 1992).

\section{REFERENCES}

Carlstrom, J.E., Plambeck, R.L., \& Thornton, D.D. 1985, IEEE Trans MTT 33, 610 .

Cooper, B.F.C. 1970, AustJ. Ph, 23, 521.

Leighton, R.B. 1978, Final Technical Report: National Science Foundation Project AST 73-04708

Padin, S., \& Ortiz, G.G. 1991, IEEE Trans. MTT 39, 7, 1239.

Padin, S., \& Ewing, M.S. 1989, IEEE Trans. IM, IM-38, 1109.

Padin, S., Clark, T.S., Ewing, M.S., Finch, R.P., Lawrence, R.P., Navarro, J., Scott, S.L., Scoville, N.Z., Seeliger, C., \& Seling, T.V. 1992, IEEE Trans., (in press).

Padin, S., \& Soares, S. 1992 in "Millimeter and Submillimeter Interferometry", $A S P$ Conference Series, in press.

Padin, S., Scott, S.L., Woody, D.P., Scoville, N.Z., Seling, T.V., Finch, R.P., Giovanine, C.J., \& Lawrence, R.P. 1991, PASP, 103, 461.

Padin, S., Woody, D.P., \& Scott, S.L. 1988, Radio Science, 23, 1067.

Rand, R. \& Kulkarni, S.R. 1990, ApJ, 349, L43.

Sargent, A.I. \& Beckwith, S.V.W. 1991, ApJ, 382, L31.

Scott, S.L. \& Woody, D.P. 1991, in IAU Colloquium 131, A.S.P. Conference Series vol 19, eds T.J. Cornwell \& R.A. Perley, 119. 
Scott, S.L. \& Finch, R.P. 1992 in "Proceedings of Workshop for Remote Observing", in press.

Scoville, N.Z. 1992 in "Millimeter and Submillimeter Interferometry", A.S.P. Conference Series, in press.

Scoville, N.Z., Thakkar, D., Carlstrom, J.E., \& Sargent, A.I. 1993, Ap.J. (Letters), in press.

Scoville, N.Z., Carlstrom, J.E., Chandler, C.J., Phillips, T., Scott, S.L., \& Tilanus, R.P. 1993, in preparation.

Shah, K.P., Muhleman, D.O., \& Berge, G.L. 1991, Icarus, 93, 96.

Swarup, G.,\& Yang, K.S. 1961, IRE Trans. AP AP-9, 75.

Wilson, C.D. \& Scoville, N.Z. 1990, ApJ, 347, 743.

Woody, D.P. 1990 in International Symposium on Millimeter and Submillimeterwave Astronomy, ed. G.D. Watt \& A.S. Webster (Dodrecht:Kluwer), 43.

Woody, D.P., Giovanine, C.J., \& Miller, R.E. 1989, IEEE Trans. MAG, MAG$25,1366$.

\section{DISCUSSION}

R. Crutcher Is your dual polarization system set up to do line and continuum polarimetry?

N. Scoville The polarization measurements done this season will be done switching a $1 / 4$ wave plate (ie non-simultaneous) and probably next season we will have 2 mixers operating in pair bound for simultaneous polarization measurements. For both configurations the continuum and spectral line measurements are made simultaneously. 